Kai-Hui Li* and Chong Liu

\title{
The crystal structure of catena-poly[( $\mu_{2}-4-($ benzo[d]imidazol-2-yl) benzenecarboxylato- $\left.\mathrm{\kappa}^{2} \mathrm{~N}, 0\right)$ - $\left(\mu_{2}-4\right.$-(benzo[d]imidazol-2-yl) benzenecarboxylato- $\left.\mathrm{K}^{3} \mathrm{~N}, \mathrm{O}: \mathrm{O}^{\prime}\right)$ cadmium(II)]dihydrate, $\mathrm{C}_{28} \mathrm{H}_{22} \mathrm{CdN}_{4} \mathrm{O}_{6}$
}

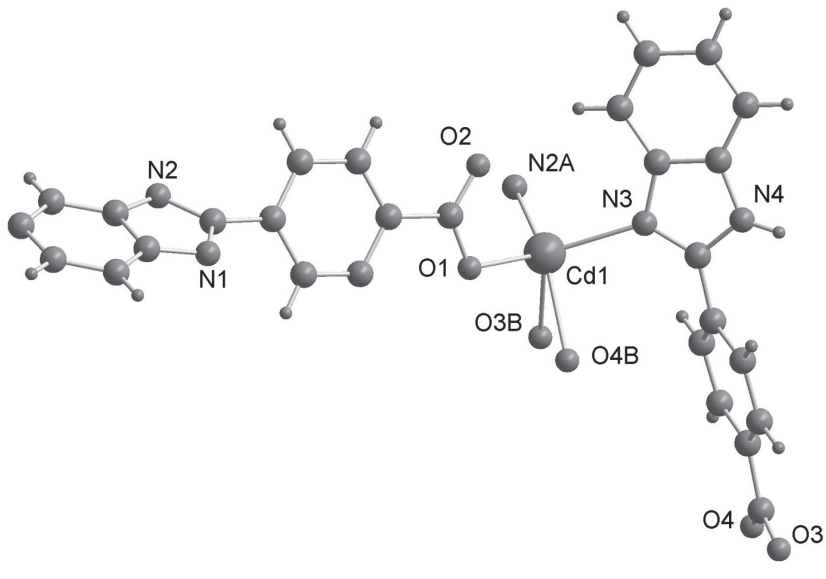

https://doi.org/10.1515/ncrs-2020-0149

Received March 19, 2020; accepted June 2, 2020; available online July 14,2020

\begin{abstract}
$\mathrm{C}_{28} \mathrm{H}_{22} \mathrm{CdN}_{4} \mathrm{O}_{6}$, triclinic, $P \overline{1} \quad$ (no. 2), $a=8.8463$ (3) $\AA$, $b=9.0275(3) \AA, \quad c=15.6752(5) \AA, \quad \alpha=78.033(3)^{\circ}$, $\beta=88.988(3)^{\circ}, \quad \gamma=84.843(3)^{\circ}, \quad V=1219.66(7) \AA^{3}, \quad Z=2$, $R_{\mathrm{gt}}(F)=0.0333, w R_{\text {ref }}\left(F^{2}\right)=0.0732, T=291 \mathrm{~K}$.
\end{abstract}

\section{CCDC no.: 2007141}

A part of the polymeric structure is shown in the figure $(\mathrm{A}=1-x, 1-y, 1-z ; \mathrm{B}=2-x, 1-y,-z)$. Table 1 contains crystallographic data and Table 2 contains the list of the atoms including atomic coordinates and displacement parameters.

*Corresponding author: Kai-Hui Li, School of Environmental and Municipal Engineering, North China University of Water Resources and Electric Power, Zhengzhou, Henan 450045, P.R. China; and Henan Key Laboratory of Water Enviroment Simulation and Treatment, North China University of Water Resources and Electric Power, Zhengzhou, Henan 450045, P.R. China, e-mail: likaihui@ncwu.edu.cn

Chong Liu: School of Environmental and Municipal Engineering, North China University of Water Resources and Electric Power, Zhengzhou, Henan 450045, P.R. China; and Henan Key Laboratory of Water Enviroment Simulation and Treatment, North China University of Water Resources and Electric Power, Zhengzhou, Henan 450045, P.R. China
Table 1: Data collection and handling.

\begin{tabular}{ll}
\hline Crystal: & Colourless block \\
Size: & $0.21 \times 0.20 \times 0.18 \mathrm{~mm}$ \\
Wavelength: & Mo $K \alpha$ radiation $(0.71073 \AA)$ \\
$\mu:$ & $0.95 \mathrm{~mm}^{-1}$ \\
Diffractometer, scan mode: & SuperNova, $\omega$ \\
$\theta_{\text {max }}$, completeness: & $28.4^{\circ},>99 \%$ \\
$N(h k l)_{\text {measured }}, N(h k l)_{\text {unique }}, R_{\text {int }}:$ & $14591,5202,0.033$ \\
Criterion for $I_{\text {obs }}, N(h k l)_{\text {gt }}:$ & $I_{\text {obs }}>2 \sigma\left(I_{\text {obs }}\right), 4658$ \\
$N(\text { param })_{\text {refined }}:$ & 352 \\
Programs: & CrysAlis \\
\hline
\end{tabular}

Table 2: Fractional atomic coordinates and isotropic or equivalent isotropic displacement parameters $\left(\AA^{2}\right)$.

\begin{tabular}{lrrrr}
\hline Atom & $\boldsymbol{x}$ & $\boldsymbol{y}$ & $\boldsymbol{z}$ & $\boldsymbol{U}_{\text {iso }} / \boldsymbol{U}_{\text {eq }}$ \\
\hline Cd1 & $0.68235(2)$ & $0.33201(2)$ & $0.24244(2)$ & $0.02708(8)$ \\
O1 & $0.5787(3)$ & $0.5241(2)$ & $0.29394(14)$ & $0.0497(6)$ \\
O2 & $0.4117(2)$ & $0.3628(3)$ & $0.34559(16)$ & $0.0509(6)$ \\
O3 & $1.0552(2)$ & $0.6371(2)$ & $-0.22212(13)$ & $0.0427(5)$ \\
O4 & $1.2161(2)$ & $0.4731(2)$ & $-0.13856(13)$ & $0.0367(5)$ \\
N1 & $0.1874(3)$ & $1.0363(2)$ & $0.52732(15)$ & $0.0333(5)$ \\
H1 & 0.1746 & 1.0729 & 0.4725 & $0.040^{*}$ \\
N2 & $0.2535(2)$ & $0.8724(2)$ & $0.64975(14)$ & $0.0268(5)$ \\
N3 & $0.5776(2)$ & $0.2362(2)$ & $0.13622(14)$ & $0.0271(5)$ \\
N4 & $0.4855(2)$ & $0.2063(2)$ & $0.01061(15)$ & $0.0304(5)$ \\
H4 & 0.4764 & 0.2131 & -0.0446 & $0.036^{*}$ \\
C1 & $0.4072(3)$ & $0.5927(3)$ & $0.39922(17)$ & $0.0294(6)$ \\
C2 & $0.4613(3)$ & $0.7350(3)$ & $0.38981(19)$ & $0.0381(7)$ \\
H2 & 0.5338 & 0.7647 & 0.3475 & $0.046 *$ \\
C3 & $0.4082(3)$ & $0.8320(3)$ & $0.44258(19)$ & $0.0382(7)$ \\
H3 & 0.4418 & 0.9287 & 0.4339 & $0.046^{*}$ \\
C4 & $0.3051(3)$ & $0.7878(3)$ & $0.50887(17)$ & $0.0280(6)$ \\
C5 & $0.2548(3)$ & $0.6439(3)$ & $0.52011(19)$ & $0.0333(6)$ \\
H5 & 0.1871 & 0.6117 & 0.5647 & $0.040^{*}$ \\
C6 & $0.3050(3)$ & $0.5482(3)$ & $0.46520(19)$ & $0.0336(6)$ \\
H6 & 0.2695 & 0.4524 & 0.4728 & $0.040^{*}$ \\
C7 & $0.4669(3)$ & $0.4847(3)$ & $0.34235(19)$ & $0.0362(7)$ \\
C8 & $0.2492(3)$ & $0.8948(3)$ & $0.56325(17)$ & $0.0270(6)$ \\
C9 & $0.1488(3)$ & $1.1112(3)$ & $0.59319(18)$ & $0.0289(6)$ \\
C10 & $0.0818(3)$ & $1.2566(3)$ & $0.5932(2)$ & $0.0382(7)$ \\
H10 & 0.0519 & 1.3236 & 0.5416 & $0.046^{*}$ \\
C11 & $0.0619(3)$ & $1.2969(3)$ & $0.6724(2)$ & $0.0424(8)$ \\
H11 & 0.0160 & 1.3927 & 0.6749 & $0.051^{*}$ \\
C12 & $0.1089(4)$ & $1.1973(3)$ & $0.7493(2)$ & $0.0422(7)$ \\
H12 & 0.0970 & 1.2295 & 0.8018 & $0.051^{*}$ \\
C13 & $0.1726(3)$ & $1.0521(3)$ & $0.74954(19)$ & $0.0358(7)$ \\
& & & &
\end{tabular}


Table 2 (continued)

\begin{tabular}{lrrrr}
\hline Atom & $\boldsymbol{x}$ & $\boldsymbol{y}$ & $\boldsymbol{z}$ & $\boldsymbol{U}_{\text {iso }}{ }^{*} \boldsymbol{U}_{\text {eq }}$ \\
\hline H13 & 0.2021 & 0.9856 & 0.8013 & $0.043^{*}$ \\
C14 & $0.1914(3)$ & $1.0087(3)$ & $0.67009(17)$ & $0.0273(6)$ \\
C15 & $0.9544(3)$ & $0.4520(3)$ & $-0.10898(17)$ & $0.0273(6)$ \\
C16 & $0.8041(3)$ & $0.4979(3)$ & $-0.13176(19)$ & $0.0345(7)$ \\
H16 & 0.7812 & 0.5704 & -0.1823 & $0.041^{*}$ \\
C17 & $0.6881(3)$ & $0.4363(3)$ & $-0.07966(19)$ & $0.0343(7)$ \\
H17 & 0.5877 & 0.4663 & -0.0960 & $0.041^{*}$ \\
C18 & $0.7206(3)$ & $0.3296(3)$ & $-0.00280(17)$ & $0.0271(6)$ \\
C19 & $0.8716(3)$ & $0.2827(3)$ & $0.01944(18)$ & $0.0294(6)$ \\
H19 & 0.8950 & 0.2108 & 0.0702 & $0.035^{*}$ \\
C20 & $0.9862(3)$ & $0.3426(3)$ & $-0.03363(18)$ & $0.0297(6)$ \\
H20 & 1.0867 & 0.3093 & -0.0188 & $0.036^{*}$ \\
C21 & $1.0833(3)$ & $0.5245(3)$ & $-0.16039(18)$ & $0.0315(6)$ \\
C22 & $0.5956(3)$ & $0.2608(3)$ & $0.04967(17)$ & $0.0263(6)$ \\
C23 & $0.3900(3)$ & $0.1381(3)$ & $0.07347(18)$ & $0.0294(6)$ \\
C24 & $0.2655(3)$ & $0.0576(3)$ & $0.0684(2)$ & $0.0379(7)$ \\
H24 & 0.2299 & 0.0439 & 0.0154 & $0.045^{*}$ \\
C25 & $0.1976(3)$ & $-0.0009(4)$ & $0.1459(2)$ & $0.0463(8)$ \\
H25 & 0.1143 & -0.0566 & 0.1454 & $0.056^{*}$ \\
C26 & $0.2501(4)$ & $0.0207(4)$ & $0.2251(2)$ & $0.0467(8)$ \\
H26 & 0.1993 & -0.0187 & 0.2760 & $0.056^{*}$ \\
C27 & $0.3753(3)$ & $0.0990(3)$ & $0.23027(19)$ & $0.0372(7)$ \\
H27 & 0.4102 & 0.1124 & 0.2835 & $0.045^{*}$ \\
C28 & $0.4473(3)$ & $0.1572(3)$ & $0.15250(18)$ & $0.0277(6)$ \\
05 & $0.1269(3)$ & $1.2499(3)$ & $0.37493(14)$ & $0.0538(6)$ \\
H5A & 0.0962 & 1.2645 & 0.3226 & $0.081^{*}$ \\
H5B & 0.2111 & 1.2884 & 0.3641 & $0.081^{*}$ \\
O6 & $0.4477(3)$ & $0.2081(3)$ & $-0.16039(15)$ & $0.0621(7)$ \\
H6A & 0.3580 & 0.1708 & -0.1462 & $0.093^{*}$ \\
H6B & 0.4354 & 0.2988 & -0.1897 & $0.093^{*}$ \\
\hline & & & &
\end{tabular}

\section{Source of material}

A mixture of $\mathrm{Cd}\left(\mathrm{CH}_{3} \mathrm{COO}\right)_{2} \cdot 2 \mathrm{H}_{2} \mathrm{O}(0.0266 \mathrm{~g}, 0.1 \mathrm{mmol})$, 4-(benzo[d]imidazol-2-yl)benzoic acid (0.0238 g, $0.1 \mathrm{mmol}$ ), and $\mathrm{H}_{2} \mathrm{O}(15 \mathrm{~mL})$ was sealed in a $25 \mathrm{~mL}$ Teflon-lined stainless steel vessel and heated at $120{ }^{\circ} \mathrm{C}$ for 4 days. The colorless block crystals of the title compound were obtained, which were washed with water and dried in air with a $44 \%$ yield.

\section{Experimental details}

The hydrogen atoms were assigned with common isotropic displacement factors $U_{\text {iso }}(\mathrm{H})=1.2$ times $U_{\text {eq }}(\mathrm{C})$ and $U_{\text {eq }}(\mathrm{N})$, refined with riding coordinates, $U_{\text {iso }}(\mathrm{H})=1.5$ times $U_{\text {eq }}(\mathrm{O})$, refined as rotating group.

\section{Comment}

Coordination polymer (CPs), a fascinating kind of crystalline solides have drawn attention in the past decades because of their porosity, tunable frameworks, and application in many fields [4-7]. Up to now various techniques have been developed to bulid CPs due to the aesthetic appeal of their frameworks and topologies. A number of CPs have been assembled from metal centers with aromatic carboxylates since they can provide the structural rigidity and give diverse coordination modes for the generation of CPs chains, layers, or networks [8-10]. On the other hand, N-donor coligands can be incorporated, thereby enhancing the structural scope and imparting additional multi-functional behavior [11-13]. Recently, we have been interested in the construction of CPs bearing bifunctional ligands with aromatic carboxylates and $N$ heterocyclics present together [14-16]. As an extension of this project, we herein report the title structure.

The title compound exhibits one dimensional chain structure. The asymmetric unit of the title structure contains two 4-(benzo[d]imidazol-2-yl)benzoate anions, one Cd(II) ion, and two lattice water molecules. As shown in the figure, Cd1 is five-coordinated by three oxygen atoms from two different 4-(benzo[d]imidazol-2-yl)benzoate ligands and two nitrogen atoms from two other ligands. The coordination geometry of Cd1 can be described as a distorted trigonal bipyramid. The Cd-O bond lengths are in the range of 2.183(2)-2.372(2) $\AA$, and the $\mathrm{Cd}-\mathrm{N}$ bond lengths are 2.266(2) and 2.275(2) A, respectively. The two 4-(benzo[d]imidazol-2-yl)benzoate ligands adopt different $\mu_{2}$ coordination modes and each Cd(II) center is coordinated by four ligands. Cd(II) cations are connected into one-dimensional chains. There are several intermolecular hydrogen bonds in the structure of the title compound. The one-dimensional chains are joined by these hydrogen bonds to form a three-dimensional framework.

\section{References}

1. Agilent Technologies: CrysAlis ${ }^{\text {PRO }}$ Software system, version 1.171.35.19. Agilent Technologies UK Ltd, Oxford, UK (2011).

2. Sheldrick, G. M.: SHELXT - Integrated space-group and crystal-structure determination. Acta Crystallogr. A71 (2015) 3-8.

3. Sheldrick, G. M.: Crystal structure refinement with SHELXL. Acta Crystallogr. C71 (2015) 3-8.

4. Miao, S.-B.; Ji, B.-M.; Wang, Y.-F.; Li, Z.-H.; Deng, D.-S.; Xu, C.-Y.; Zhou, L.: A new 3D pillared-layer porous framework with interesting open channels in the Co/triazolate/ carboxylate system: synthesis, structure and magnetism. Inorg. Chem. Commun. 62 (2015) 47-50.

5. Ma, S.; Zhou, H.-C.: Gas storage in porous metal-organic frameworks for clean energy applications. Chem. Commun. 46 (2010) 44-53.

6. Li, Z.-H.; Xue, L.-P.; Qin, Q.-P.; Zhao, Y.-J.: Synthesis, structure, and catalytic properties of a copper(II) coordination polymer material constructed from 5-nitro-1,2,3-benzenetricarboxylic acid and bis(4-pyridylformyl)piperazine mixed ligands. J. Solid State Chem. 278 (2019) 120908.

7. Wang, Z. Q.; Zheng, C. J.; Wang, W. Z.; Xu, C.; Ji, B. M.; Zhang, X. H.: Synthesis, structure, and photophysical properties of two four-coordinate $\mathrm{Cu}^{\prime}$-NHC complexes with efficient delayed fluorescence. Inorg. Chem. 55 (2016) 2157-2164. 
8. Chen, Z.-X.; Zhou, Y.-M.; Weng, L.-H.; Zhao, D.-Y.: Mixedsolvothermal syntheses and structures of six new zinc phosphonocarboxylates with zeolite-type and pillarlayered frameworks. Cryst. Growth Des. 8 (2008) 4045-4053.

9. Xue, L.-P.; Li, Z.-H.; Li, S.-H.; Wang, J.-G.: Synthesis, crystal structure and fluorescent property of a new onedimensional cadmium(II) coordination polymer based on 3,4-thiophenedicarboxylic acid and 1,10-phenanthroline. Chin. J. Struct. Chem. 32 (2013) 704-708.

10. Liu, G.-Z.; Xin, L.-Y.; Wang, L. Y.: Ancillary ligand-mediated syntheses and fluorescence properties of zinc(II) complexes based on flexible benzene dicarboxylic acid. CrystEngComm 13 (2011) 3013-3020.

11. Miao, S.-B.; Li, Z.-H.; Xu, C.-Y.; Ji, B.-M.: A new 3-fold interpenetrating 3D Zn(II) metal-organic framework: synthesis, structure and luminescent property. Chin. J. Struct. Chem. 35 (2016) 1960-1966.

12. Lan, H.-H.; Li, X.-T.; Chu, W.-J.; Xu, C.-Y.; Ji, B.-M.: Syntheses, crystal structures and luminescence properties of two $\mathrm{Zn}$ (II) coordination polymers based on flexible bisbenzimidazole ligand. Chin. J. Inorg. Chem. 35 (2019) 1896-1902.

13. Miao, S.-B.; Wang, Y.-F.; Deng, D.-S.; Xu, C.-Y.; Li, Z.-H.; Ji, B.-M.: A new Cu-based metal-organic framework built upon infinite 1D rod-shaped secondary building units. Inorg. Chem. Commun. 78 (2017) 25-27.

14. Deng, D.-S.; Kang, G.-H.; Ji, B.-M.; Li, H.-L.; Qu, G.-R.; Fan, X.-S.: Syntheses photoluminescent properties, and structural investigation of five complexes based on a new T-shaped 2(pyridin-3-yl)-4,6-pyrimidine dicarboxylic acid ligand: structure evolution from one-dimensional chains to three-dimensional architectures. Aust. J. Chem. 66 (2013) 1342-1351.

15. Miao, S.-B.; Wang, Y.-F.; Deng, D.-S.; Xu, C.-Y.; Li, Z.-H.; Ji, B.-M.: Two Co(II) coordination polymers based on 3,5-di (4H-1,2,4-triazole-4-yl)benzoic acid ligand: syntheses, structures and magnetic property. Chin. J. Struct. Chem. 37 (2018) 1102-1108.

16. Miao, S.-B.: Crystal structure of poly[aqua- $\left(\mu_{3}-3,5-\mathrm{di}(4 \mathrm{H}\right.$ 1,2,4-triazolyl-4- $\left.\mathrm{K}^{3} N, N^{\prime}: N^{\prime \prime}\right)$ benzenecarboxylato) silver(I)], $\mathrm{C}_{11} \mathrm{H}_{9} \mathrm{AgN}_{6} \mathrm{O}_{3}$. Z. Kristallogr. NCS 232 (2017) 711-712. 